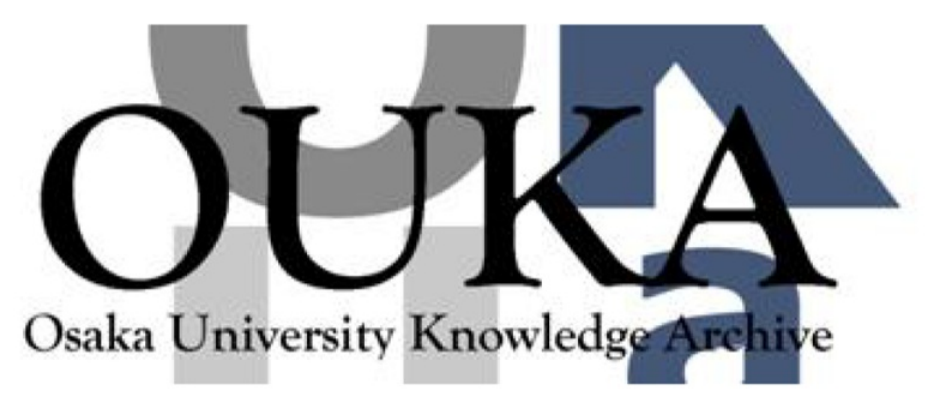

\begin{tabular}{|c|l|}
\hline Title & $\begin{array}{l}\text { Photoinduced dichroism and optical anisotropy } \\
\text { in a liquid-crystalline azobenzene side chain } \\
\text { polymer caused by anisotropic angular } \\
\text { distribution of trans and cis isomers }\end{array}$ \\
\hline Author(s) & $\begin{array}{l}\text { Blinov, Lev M.; Kozlovsky, Mikhail V.; Ozaki, } \\
\text { Masanori et al. }\end{array}$ \\
\hline Citation & Journal of Applied Physics. 84(7) p. 3860-p. 3866 \\
\hline Issue Date & $1998-10-01$ \\
\hline oaire:version & VoR \\
\hline URL & https://hdl. handle. net/11094/75854 \\
\hline rights & \\
\hline Note & \\
\hline
\end{tabular}

Osaka University Knowledge Archive : OUKA

https://ir. Library. osaka-u. ac. jp/

Osaka University 


\section{Photoinduced dichroism and optical anisotropy in a liquid-crystalline azobenzene side chain polymer caused by anisotropic angular distribution of trans and cis isomers}

Cite as: Journal of Applied Physics 84, 3860 (1998); https://doi.org/10.1063/1.368565 Submitted: 14 May 1998. Accepted: 02 July 1998. Published Online: 21 October 1998

Lev M. Blinov, Mikhail V. Kozlovsky, Masanori Ozaki, Kent Skarp, and Katsumi Yoshino

\section{ARTICLES YOU MAY BE INTERESTED IN}

Photoinduced surface deformations on azobenzene polymer films

Journal of Applied Physics 86, 4498 (1999); https://doi.org/10.1063/1.371393

Surface relief grating in azo-polymer obtained for s-s polarization configuration of the writing beams

Applied Physics Letters 101, 193301 (2012); https://doi.org/10.1063/1.4764950

Gradient force: The mechanism for surface relief grating formation in azobenzene functionalized polymers

Applied Physics Letters 72, 2096 (1998); https://doi.org/10.1063/1.121287

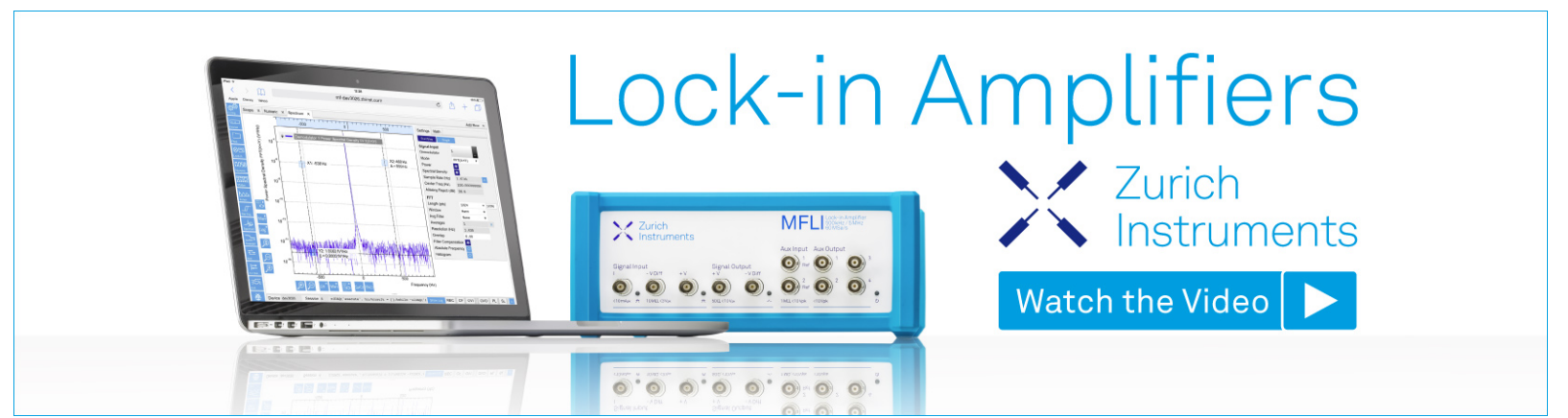




\title{
Photoinduced dichroism and optical anisotropy in a liquid-crystalline azobenzene side chain polymer caused by anisotropic angular distribution of trans and cis isomers
}

\author{
Lev M. Blinov \\ Department of Electronic Engineering, Faculty of Engineering, Osaka University, 2-1 Yamada-Oka, Siuta, \\ Osaka 565, Japan and Institute of Crystallography, Russian Academy of Sciences, \\ 117333, Leninsky prosp. 59, Moscow, Russia \\ Mikhail V. Kozlovsky \\ Institute of Crystallography, Russian Academy of Sciences, 117333, Leninsky prosp. 59, Moscow, Russia \\ and Physics Department, Chalmers University of Technology, S-41296, Göteborg, Sweden \\ Masanori Ozaki \\ Department of Electronic Engineering, Faculty of Engineering, Osaka University, 2-1 Yamada-Oka, Siuta, \\ Osaka 565, Japan \\ Kent Skarp \\ Physics Department, Chalmers University of Technology, S-41296, Göteborg, Sweden \\ Katsumi Yoshinoa) \\ Department of Electronic Engineering, Faculty of Engineering, Osaka University, 2-1 Yamada-Oka, Siuta, \\ Osaka 565, Japan
}

(Received 14 May 1998; accepted for publication 2 July 1998)

\begin{abstract}
Photochromism has been studied for two comb-like liquid-crystalline copolymers (I) and (II) containing azobenzene chromophores in their side chains. In a smectic glass phase of both copolymers, upon short-time irradiation by UV light, long-living cis isomers are observed. Both copolymers manifest the photoinduced anisotropy, the physical mechanisms of which seem to be quite different. In spin-coated films of polymer (II), the origin of the anisotropy is a strong stable dichroism, which is due to an enrichment and depletion of the chosen angular direction, correspondingly, with trans and cis isomers of the azobenzene chromophores. Polymer (I) manifests no dichroism at all, and its induced optical anisotropy may be accounted for by a rather slow chromophore reorientation. In copolymer (II) a considerable reorientation of the mesogenic groups also occurs as a secondary phenomenon at the stage of the cis isomer formation only. This observation shed more light on the general process of the light-induced molecular reorientation in polymers, liquid crystals, and Langmuir-Blodgett films, which is of great importance for holographic information recording. (C) 1998 American Institute of Physics.
\end{abstract}

[S0021-8979(98)03419-7]

\section{INTRODUCTION}

Azobenzene derivatives are well-known systems showing reversible transformations upon irradiation with UV and visible light. ${ }^{1,2}$ Typically, the absorption of a UV light quantum transforms an elongated rod-like molecular form (transisomer of the azobridge) into a bent banana-like form (cis isomer). The transformations are accompanied by dramatic changes in electronic spectra of the compounds (photochromism). Irradiation of the cis form of the same compound by a visible light causes a backward transformation of cis isomers into the trans ones, the process being typically accompanied by thermal cis-trans transitions. The photochromism of azobenzene derivatives may be observed in both dilute solutions or condensed phases of azocompounds. There have been reported various types of holographic photorecording media ${ }^{3-5}$ and photo-optical switching devices ${ }^{6}$ based on photochromic behavior.

\footnotetext{
${ }^{\text {a)} E l e c t r o n i c ~ m a i l: ~ y o s h i n o @ e l e . e n g . o s a k a-u . a c . j p ~}$
}

Recently, a number of studies were devoted to a photoinduced dichroism and a related phenomenon of the photoinduced optical anisotropy in amorphous polymers and polymer liquid crystals with azodyes introduced as dopants or chromophores chemically attached to a polymer chain. ${ }^{7-9}$ In this case, a linearly polarized light is used to induce the anisotropy and the mechanism of the phenomenon is, presumably, based on the change of the azochromophore orientational distribution function after repetitive trans-cis-trans phototransitions. There were also reports on preparation of polar nonlinear optical materials by a photoelectropoling technique, ${ }^{10,11}$ and observation of giant photoinduced optical anisotropy in Langmuir-Blodgett films (LBFs) based on amphiphilic azodyes. ${ }^{12,13}$ A recent review article ${ }^{14}$ is devoted to the phenomena originated from the photoinduced molecular orientation in polymers, liquid crystals, and LBFs.

Chiral systems attract a special interest. There were studies of the influence of irradiation on the pitch of cholesteric liquid crystals ${ }^{15,16}$ due to a change in the relative percentage 
of the cis and trans isomers and even total unwinding of cholesteric structure in a polymer liquid crystal ${ }^{17}$ caused by photoinduced chromophore reorientation. Irradiation of the $\mathrm{TGB}_{A} *$ phase of a methacrylic copolymer (I) containing an azo-side chain was shown to prohibit the phase transition to the smectic $A$ phase due, reportedly, to a change in the cisand trans-isomer ratio. ${ }^{18}$

Despite a large number of papers devoted to the photoinduced optical anisotropy in different azochromophore containing systems, the mechanism of the phenomenon is still not clear. For example, the anisotropy may be induced by a long-wave Ar-laser light, which is absorbed in the cis-isomer $n-\pi^{*}$ band and, hence, may interact only with cis isomers the amount of which is very small under ambient conditions. Detailed spectroscopic studies of the phenomena are rare (some examples are Refs. 7 and 19). It is not clear which isomers play a decisive role in the phase transformations reported in Ref. 18. The trans-cis transitions are, as a rule, rather fast, but reorientation phenomena are, on the contrary, rather slow, and it is not clear how the two kinetic processes become parallel to each other.

In an attempt to clarify these questions we have carried out spectroscopic and kinetic studies of two liquid-crystalline copolymers [one of them is copolymer (I) (Ref. 18)] in both chloroform solution and very thin spin-coated films. The latter allowed us to follow the evolution of both chromophores and mesogenic group absorption bands upon irradiation. In the course of the work we have observed a new, to our knowledge, effect when a stable photoinduced dichroism and optical anisotropy is created not by the chromophore reorientation process but due to selective, polarization-dependent depletion and enrichment of a certain angular direction with long-living cis or trans isomers. The effect has specific features that may be useful for efficient holographic grating recording.

\section{EXPERIMENT}

\section{A. Materials}

\section{Synthesis}

In the present work we studied two side chain copolymers (I) and (II) with their chemical structure shown in Fig. 1. Both copolymers contain the same mesogenic moiety (A) but different chromophores (B and C).

Chiral methacrylic comonomer (A) was synthesized according to the method described in Ref. 20. Monomer (B), was obtained according to a method reported earlier. ${ }^{21}$ The details of the copolymerization process and purification of copolymer (I) that contains $8 \mathrm{~mol} \%$ of photochromic monomer B may be found in Ref. 18.

Synthesis of monomer C ( $p$-11-methacryloyloxyundecanoyloxy- $p$ '-methoxy-azobenzene) for copolymer (II) was made via two intermediate stages (4-methoxy4 '-hydroxyazobenzene and $p$-11-bromoundecanoyloxy- $p^{\prime}$ methoxy-azobenzene) and will be described in a separate paper. Copolymerization of the corresponding methacrylic monomers was carried out in dry benzene (10\% solution) during $72 \mathrm{~h}$ at $60{ }^{\circ} \mathrm{C}$ using AIBN as the initiator (0.1 wt \%). The $0.3 \mathrm{~g}$ copolymer batch was reprecipitated three times

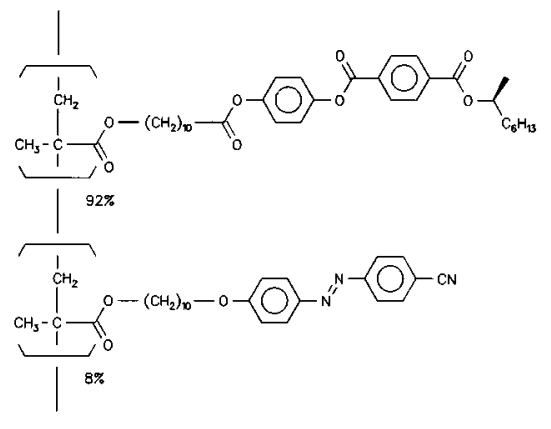

(A)

(I)

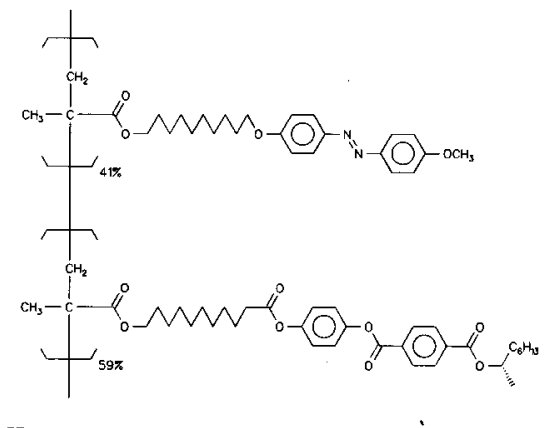

(C)

(II)

FIG. 1. Chemical structures of copolymers (I) and (II).

from chloroform to methanol and washed several times by $50 \mathrm{ml}$ of boiling methanol. The composition of the copolymer has been assumed to be equal to that of the monomer mixture due to the almost identical chemical structure of the comonomers (A:C=59:41 mol \%). The polymerization degree was 315 .

\section{Structure}

$\mathrm{X}$-ray investigations show that both materials have a smectic layered structure over a wide temperature range below the isotropic phase down to the glassy state (including room temperature).

The structural features of copolymer (I) have been described in detail. ${ }^{18}$ If the cooling is fast enough (faster than $10 \mathrm{~K} / \mathrm{min})$ a monotropic twist-grain-boundary smectic $A^{*}$ phase $\left(\mathrm{TGB}_{A^{*}}\right)$ forms from the true liquid phase $\left(T_{\mathrm{AI}}\right.$ $=53.3{ }^{\circ} \mathrm{C}$ ), the former can be frozen in the glassy state. For slow cooling a second phase transition occurs at $28{ }^{\circ} \mathrm{C}$, from the $\mathrm{TGB}_{A} *$ into a conventional $\mathrm{Sm} A *$ phase. It is of importance that irradiation of the polymer film by unpolarized light in the 400-500 $\mathrm{nm}$ wavelength range hinders the $\mathrm{TGB}_{A} *-\mathrm{Sm} A^{*}$ phase transition during slow cooling. As a result, the irradiated polymer film does not scatter light and remains completely transparent at room temperature. The transparent film becomes birefringent when it is irradiated by the linearly polarized light.

For copolymer (II), from x-ray measurements made with a setup described in Ref. 22, a layered phase starts to appear (on cooling) at around $73{ }^{\circ} \mathrm{C}$, with a layer spacing of $31.5 \AA$. The layer spacing decreases slightly with decreasing temperatures, down to about $31.2 \AA$ at $30^{\circ} \mathrm{C}$. Observation of a 


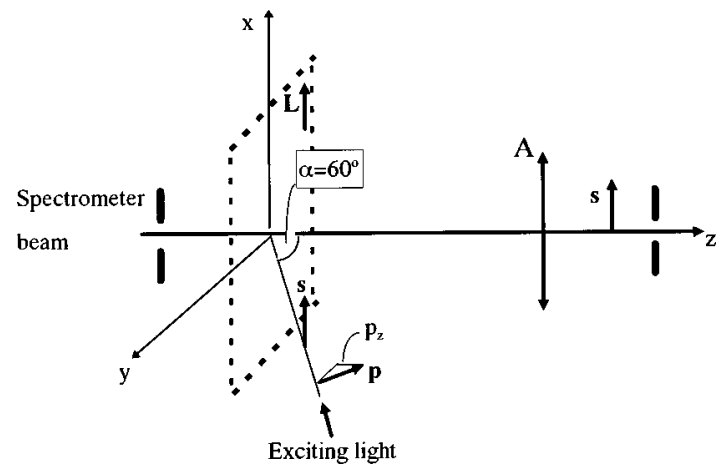

FIG. 2. Geometry of the photochromism study. The spectrometer probe beam is directed along the $z$ axis, with the analyzer transmitting vertically $(s-)$ polarized light. A quartz plate with a film is installed perpendicular to the beam and may rotate around the $z$ axis, forming an angle $\varphi$ between the long axis of plate $\mathbf{L}$ and the $x$ direction. The incident plane of the UV or visible exciting beam coincides with the $y-z$ (horizontal) plane, the angle of incidence is $60^{\circ}$, and the electric light vector may be either $\mathbf{s}$ or $\mathbf{p}$.

thick polymer film under crossed polarizers does not reveal any texture resembling typical liquid-crystalline textures of nematic and smectic phases. Only two textures with a very weak contrast observed are: the one after illumination (smooth with very small 1-4 $\mu \mathrm{m}$ blurred spots) and the other attained without illumination (scattering texture with a faint, blurred blue-green color). Therefore, most probably, copolymer (II) also shows a TGB phase, as in the case of other similar systems. ${ }^{18,23}$

\section{Films}

Spectral measurements of photochromism were made on both the chloroform solutions and thin (about $100 \mathrm{~nm}$ ) spincoated films. The films were prepared from 50:1 (by weight) chloroform solutions of the copolymers and deposited onto quartz substrates with two subsequent speeds, $500 \mathrm{rpm}(10 \mathrm{~s})$ and $1700 \mathrm{rpm}(30 \mathrm{~s})$. The light-induced birefringence was studied under a polarizing microscope using thicker layers of the materials (few $\mu \mathrm{m}$ ) confined between glass plates.

\section{B. Measurements}

\section{Photochromism}

Photochromism measurements were carried out with Hewlett-Packard diode array spectrometer (model HP $8452 \mathrm{~A})$. The spectrometer allowed for recording a spectrum for $3 \mathrm{~s}$, therefore, the kinetics of photoisomerization could be studied. Irradiation of the samples was made directly in the spectrometer compartment using a $100 \mathrm{~W}$ metal-halide lamp IMH-160 (Sigma Koki) supplied with a quartz light guide. To induce photoisomerization, a UV-pass (visible-cut) filter was installed which provided about $20 \mathrm{~mW} / \mathrm{cm}^{2}$ light power in the range 300-400 nm. $1 \mathrm{~cm}$ quartz cells with chloroform solution of a copolymer were illuminated from their open top.

\section{Photoinduced dichroism}

The geometry of our experiments is shown in Fig. 2. The samples were installed at the spectrometer light-emitting slit normally to the probing beam. Polarization absorption mea-

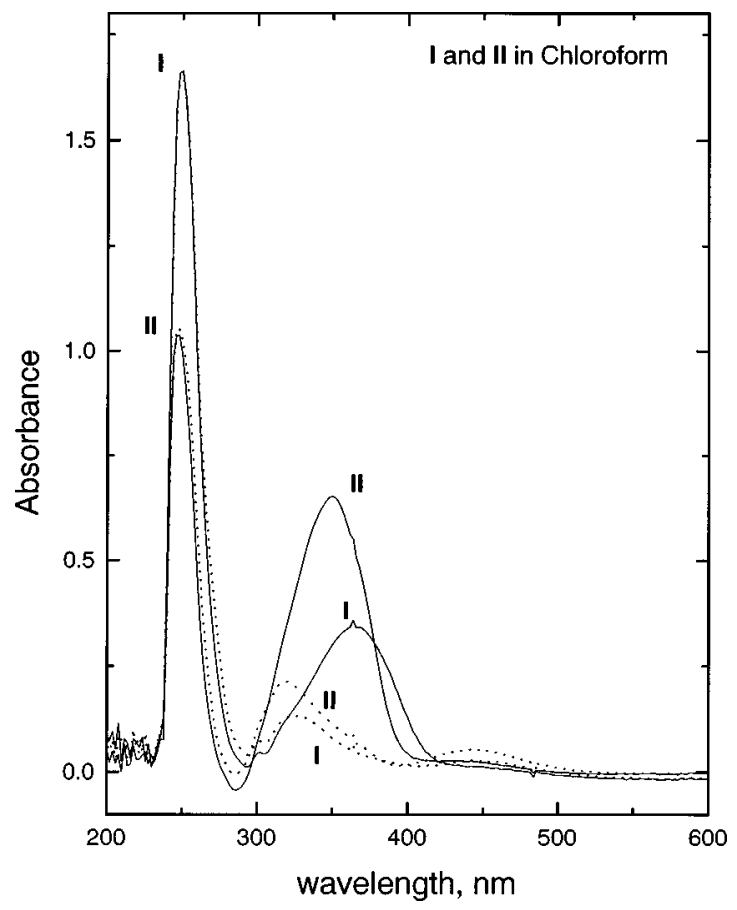

FIG. 3. Virgin spectra of copolymers (I) and (II) in chloroform solutions (trans form, solid lines) and spectra of the solutions irradiated by UV light for $30 \mathrm{~s}$ (cis form, dotted lines). Concentrations of copolymers I and II are 140 and $60 \mathrm{mg} / \mathrm{L}$, respectively.

surements were made with a Glan prism analyzer installed at the light-receiving slit of the spectrophotometer. The plane of incidence of the exciting light (UV or visible) was horizontal and the incidence angle $\alpha$ was about $60^{\circ}$ with respect to the cell normal. A polarizing film was installed after a corresponding filter and allowed us to obtain both $s$ - or $p$ polarized exciting beams.

\section{Photoinduced optical anisotropy}

For birefringence measurements at various temperatures, samples were installed into a heating stage of a Mettler device and studied under a polarizing microscope. The strong light of the microscope itself with proper filters was used to induce anisotropy. The images were recorded with chargecoupled device camera and tape recorder.

\section{RESULTS AND DISCUSSION}

\section{A. Photochromism in solutions}

Virgin spectra of copolymers (I) and (II) are shown in Fig. 3 by solid lines I and II, respectively. In both cases, we see a characteristic band belonging to mesogenic fragment $\mathrm{A}$ at $\lambda \approx 250 \mathrm{~nm}$ and a band belonging to chromophores $\mathrm{B}$ and $\mathrm{C}$ in their trans state, at $\lambda \approx 366 \mathrm{~nm}$ (I) and $350 \mathrm{~nm}$ (II). The difference in mesogen peak heights comes from different concentrations of solution, and the difference in relative heights of the mesogen and chromophore bands is due to the smaller part of the chromophore units in polymer (I). In addition, there is a weak shoulder at the long-wave slope of the trans-isomer band, which is usually referred to as an $n-\pi^{*}$ transition in cis isomers ${ }^{24}$ since this transition is forbidden by symmetry for the trans isomers. Irradiation with UV light 


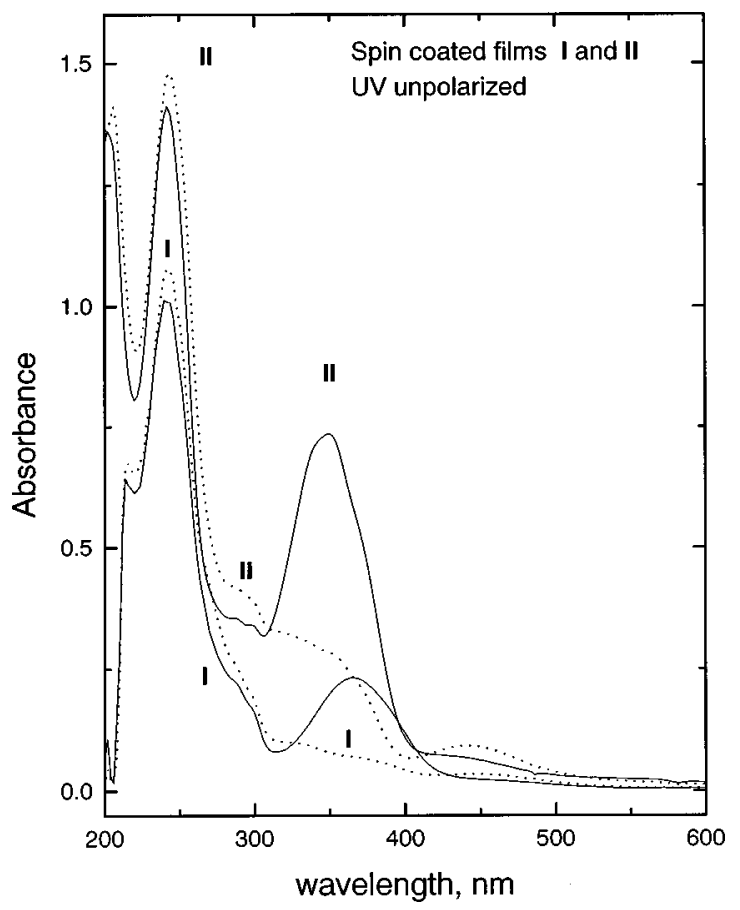

FIG. 4. Virgin spectra of spin-coated films of copolymers (I) and (II) (trans form, solid lines) and spectra of the films irradiated by UV light for $3 \mathrm{~min}$ for (I) and $10 \mathrm{~min}$ (II) (cis form, dotted lines).

strongly suppresses the trans bands and increases absorption in the range of $250-300 \mathrm{~nm}\left(\pi-\pi^{*}\right.$ transitions in cis isomers) and in the range $400-500 \mathrm{~nm}$, where a maximum forms corresponding to the $n-\pi^{*}$ transitions in cis isomers [448 $\mathrm{nm}$ for (I) and $442 \mathrm{~nm}$ for (II)]. Note that the height of the maximum of the mesogen absorption at $250 \mathrm{~nm}$ is almost insensitive to the trans-cis transitions in chromophores.

The cis form of the two azopolymers is extremely stable in comparison with other azocompounds. ${ }^{25}$ In polymer (I) after $24 \mathrm{~h}$ relaxation in darkness the trans-absorption band was restored to about $80 \%$ of its initial height, but additional illumination with yellow or green light restored it for $10 \mathrm{~s}$ even to a level exceeding the initial one some $5 \%$. Even the slower dark relaxation of the cis isomers is observed for polymer (II): for $55 \mathrm{~h}$ the trans band was restored only to some $20 \%-30 \%$ of the initial height, but went back to $100 \%$ in $30 \mathrm{~s}$ upon illumination with strong yellow light. Quite curiously, the stable cis form even allows preparation of cast films directly from UV irradiated solutions; after evaporation of chloroform the films keep the acquired cis form for many days under ambient conditions.

\section{B. Photochromism of spin-coated films}

Figure 4 shows photochromism spectra for copolymers (I) and (II) in the form of thin film spin-coated on quartz substrates. Solid curves show spectra of the films before irradiation; dotted curves are taken after irradiation of the films by unpolarized UV light for $3 \mathrm{~min}$ [copolymer (I)] and $10 \mathrm{~min}$ [copolymer (II)]. The basic features of the spectra are the same as for chloroform solutions: on irradiation, the trans band (367 and $348 \mathrm{~nm}$ in I and II, respectively) is suppressed, the cis absorption (at 260-300 nm) increases,

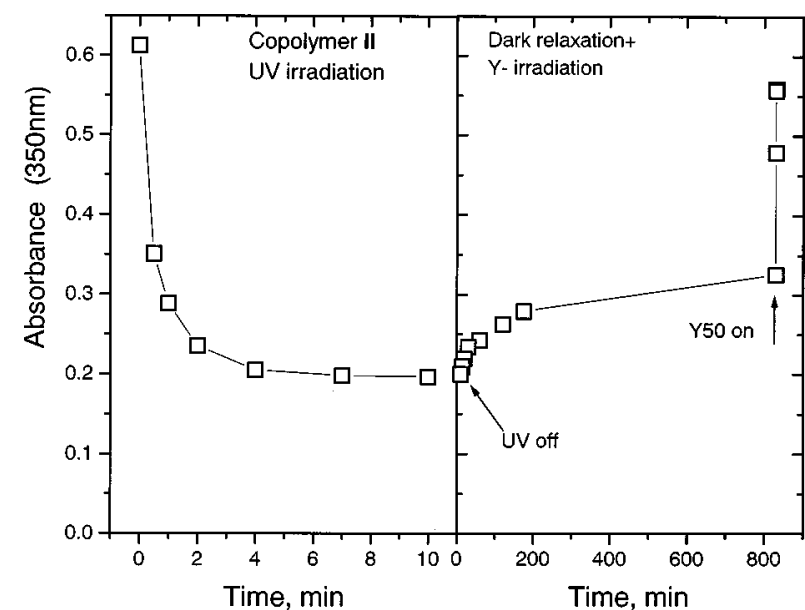

FIG. 5. Time evolution of the trans-form absorption maximum $(\lambda$ $=350 \mathrm{~nm}$ ) in a spin-coated film of copolymer (II) upon UV irradiation, during relaxation in darkness and upon irradiation of the film by yellow light.

and the $n-\pi^{*}$ band around $440 \mathrm{~nm}$ forms. The only difference is that now mesogenic bands are sensitive to the transcis transitions; upon irradiation the absorption at $242 \mathrm{~nm}$ increases by $5 \%-7 \%$. This cannot be accounted for by growth of a cis-band pedestal under the mesogen absorption band since we do not observe such an increase of $242 \mathrm{~nm}$ absorption in the chloroform solution. The phenomenon is related to molecular reorientation and will be discussed in the next section.

The stability of the cis form in a solid state is surprisingly high especially for copolymer (II). The kinetic curve in Fig. 5 shows how the trans-form maximum $(\lambda=350 \mathrm{~nm})$ evolves with UV irradiation, during relaxation in darkness and after irradiation of the film by yellow light. First, the UV-on stage lasts about $4 \mathrm{~min}$, and subsequent relaxation in darkness, at the beginning, is fairly fast but slows down after the first $2 \mathrm{~h}$. It took more than $13 \mathrm{~h}$ to convert only $20 \%-$ $30 \%$ of cis isomers back into the trans form. Then, on irradiation with strong yellow light the trans form is restored almost completely for $1 \mathrm{~min}$. The dark relaxation time estimated for the cis isomers in films of copolymer (I) is also rather long, about $150 \mathrm{~min}$. For comparison, the cis form of low-molecular mass azodye $\mathrm{D}^{*} 5$, showing similar photochromic behavior in a chloroform solution, survives in different liquid-crystalline phases for only $1-10 \mathrm{~min} .{ }^{25}$ In the other azodyes this lifetime might even be shorter.

\section{Polarized light-induced dichroism}

It is essential that at room temperature both copolymers are in their transparent, optically isotropic, glassy state without any sign of some preferable orientation. The virgin spincoated films show no dichroism. When spin-coated films of copolymer (II) are irradiated by polarized UV light the angular distribution of cis and trans isomers becomes anisotropic: in the direction of the UV light electric vector e there appears "a polarization hole" for the trans isomers and the same direction becomes almost solely populated by cis isomers. Now the film has to be strongly dichroic. If one shines 


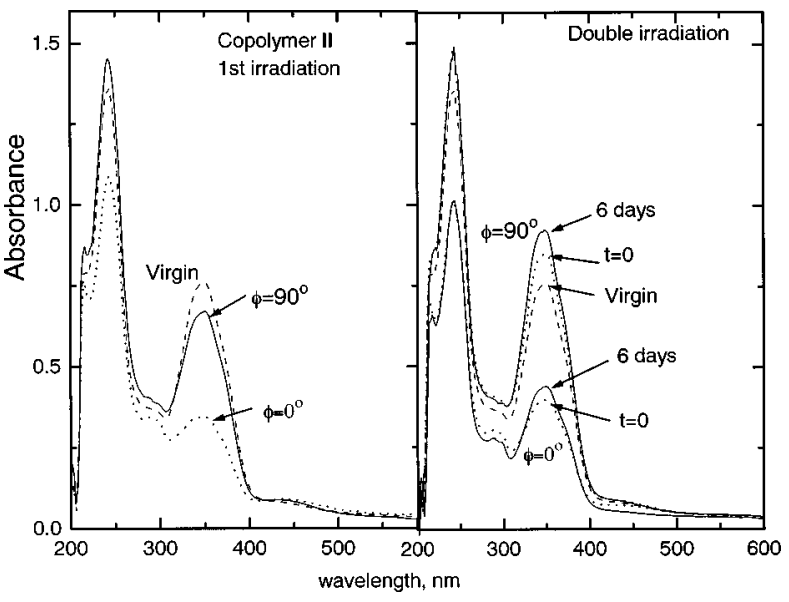

FIG. 6. Polarized absorption spectra of a spin-coated film of copolymer (II). Dashed lines on both sides indicate the virgin spectrum (no dichroism). Left side: the film is irradiated once with s-polarized UV light for $15 \mathrm{~min}$ and the spectra is taken with $s$-polarized spectrometer light for the two angular positions of the film indicated in the figure. Right side: the film was additionally irradiated with $s$-polarized yellow light for $5 \mathrm{~min}$ and the spectra of the film were taken just after the second irradiation (dotted lines) and six days later (solid lines).

yellow $\mathbf{e}_{Y}$ polarized light onto a virgin sample, the $\mathbf{e}_{Y}$ direction becomes only slightly more populated by trans isomers since the overwhelming majority of azochromophores were already in the trans form in a virgin sample. To obtain the maximum dichroic ratio we must combine the two processes, i.e., first to shine the UV light with one polarization $\mathbf{e}_{\mathrm{UV}}$ and then the yellow light with perpendicular polarization, $\mathbf{e}_{\mathrm{Y}} \perp \mathbf{e}_{\mathrm{UV}}$.

Figure 6 shows the result of such an experiment performed on a spin-coated film of copolymer (II). The initial spectrum is marked as "virgin." At first, the sample was oriented as shown in Fig. 2 with $\mathbf{L} \| x$ (position $\varphi=0^{\circ}$ ) and irradiated with $s$-polarized UV light for $15 \mathrm{~min}$ (in this case the light intensity is about $3 \mathrm{~mW} / \mathrm{cm}^{2}$ ). After this, an absorption spectrum was taken with an s-polarized spectrometer beam. Then, the sample was turned around its normal through $\varphi=90^{\circ}$ into position $\mathbf{L} \| y$ and another spectrum was taken with the same $s$ polarization. On the left side of Fig. 6 the dotted line $\left(\varphi=0^{\circ}\right)$ and the solid line $\left(\varphi=90^{\circ}\right)$ curves show the dichroism obtained after the first, UV illumination. Then, in the second $\left(\varphi=90^{\circ}\right)$ position the sample was illuminated for $5 \mathrm{~min}$ by $s$-polarized yellow light. Thus, both exciting beams were $s$ polarized and did not contain a lightfield projection onto a sample normal. On the right side of Fig. 6 dotted lines indicate that the film becomes even more strongly dichroic: for a fixed $s$ polarization of the spectrometer beam we observe quite different spectra for the two angular positions of the sample, typical cis for $\varphi=0^{\circ}$ and typical trans for $\varphi=90^{\circ}$. What is of paramount importance is an enhanced stability of the state obtained as explained above: we have observed only a slight change in the dichroism for six days under ambient conditions, the spectra for the six days old sample are shown by solid lines on the right side of Fig. 6.

Figure 6 also shows a remarkable specific feature related to the mesogen absorption band $(244 \mathrm{~nm})$, which sheds light on the process of molecular reorientation and light-induced anisotropy. On UV irradiation in the $\mathbf{L} \| x$ position $\left(\varphi=0^{\circ}\right)$ when cis isomers are created, the absorption of the mesogenic unit along the same direction significantly decreases, but for the perpendicular direction it slightly increases (left side of Fig. 6). This asymmetry means that due to formation of cis isomers there is a substantial UV-induced reorientation of the mesogenic groups in the third $z$ direction (along the normal). After the second irradiation in the $\varphi=90^{\circ}$ position by yellow light (right side) the mesogen absorption increases again, but even more slightly, since this direction is already enriched by trans isomers. It means that formation of trans isomers along one, e.g., the $y$ direction, "calls back" a small amount of the mesogenic groups from the other two ( $x$ and $z$ ) directions. Since the forward and back processes are not equally efficient, the repeated trans-cis-trans transitions may result in the collective reorientation of the mesogenic groups (and, probably, chromophores themselves) mostly at the stage of the cis isomer formation. It is of interest that the dark cis-trans relaxation seen on the right side (solid lines) does not change the mesogen absorption. Therefore, the orientation of the mesogenic groups and the relevant part of the optical anisotropy has to remain stable.

The total picture can be viewed as follows: the rather fast photoinduced formation of cis isomers exerts a strong torque on the surrounding mesogenic units, but the slow dark relaxation exerts a much weaker (if any) torque on the mesogenic groups; on the other hand, the photoinduced formation of trans isomers restores, at least partially, the initial situation with a higher ordering of mesogenic groups. This might be a key observation for understanding the general phenomenon of the photoinduced anisotropy in terms of the molecular-field model discussed in Ref. 26.

We have made additional experiments with a $p$-polarized UV beam at an incidence angle of $60^{\circ}$ (refraction angle about $35^{\circ}$ ) when the $z$ projection of the light intensity, proportional to $E_{z}^{2}$ onto the film normal is about $30 \%$ of the total intensity. This has been done in the following way: first, we irradiated a film by $s$-polarized UV light long enough $(1.5 \mathrm{~h})$ to reach the saturation close to the state shown in the left part of Fig. 6. The spectra were measured with the same $s$ polarization. When a considerable amount of $c$ is isomers had been accumulated and the mesogen absorption band was reduced dramatically, we turned the UV polarizer through $90^{\circ}$ to have only $p$-polarized UV light. The trans band starts to recover slowly (some $30 \%$ for $25 \mathrm{~min}$ ), but the mesogen absorption increased much faster and recovered completely for $15 \mathrm{~min}$. The subsequent rather fast dark relaxation results in considerable degradation of the chromophore dichroism. These observation showed that the $z$ projection of the UV light field is very essential because by producing new cis isomers this projection "calls back" the reoriented mesogenic groups much more efficiently than the in-plane field of the yellow light which creates trans isomers. For the same reason, the obliquely incident unpolarized light causes a slight increase in the mesogen absorption band shown in Fig. 4.

Surprisingly, with the same technique we were not able to induce a photodichroism in copolymer (I) in spite of the 


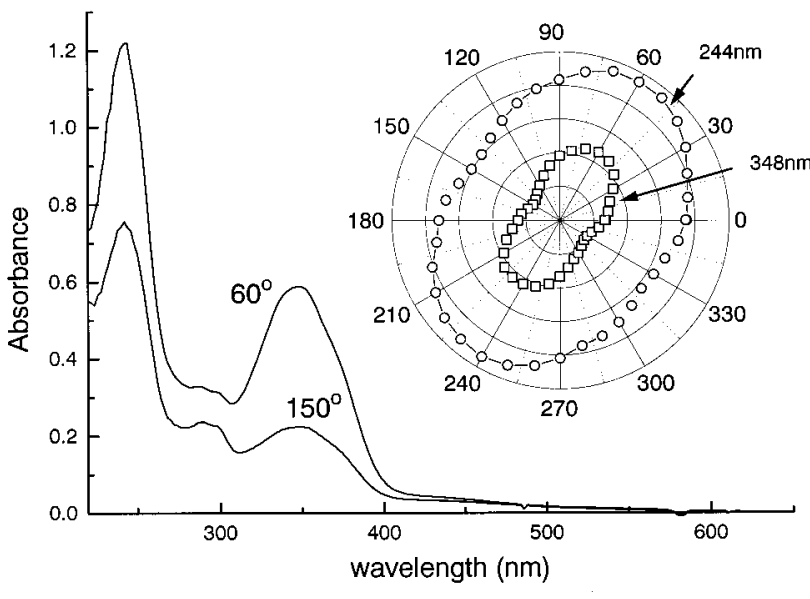

FIG. 7. Main plot: The absorption spectra of a copolymer (II) film with a previously recorded dichroism as in Fig. 6(left) after a second irradiation by UV light with the electric vector at $150^{\circ}$ with respect to the $x$ axis. Spectra are taken for two positions of the sample perpendicular to each other $\left(150^{\circ}\right.$ and $\left.60^{\circ}\right)$. Inset: the angular dependence of absorbance $(A)$ for the mesogenic band $(244 \mathrm{~nm})$ and the trans band of the azochromophore $(348 \mathrm{~nm})$. The radius of the external circle corresponds to $A=1.25$.

fact that long-lived cis isomers form easily. What we did observe is a similar decrease, as in copolymer (II), of the mesogen absorption band when we created cis isomers with the $s$-polarized UV light (with unpolarized light we had an increase of that, Fig. 4). Therefore, mesogenic groups are reorienting during the cis-isomer formation even in the copolymer with only $8 \%$ of azochromophores. The absence of the induced dichroism may be accounted for assuming much more rotational freedom for the chromophores in copolymer (I) than in (II), presumably, due to the different phase structure of the two copolymers in our spin-coated films. Unfortunately, detailed structural data are not available yet even for bulk samples.

The light-induced dichroism discussed above may be rewritten by polarized UV light with an electric vector oriented at an arbitrary angle with respect to $\mathbf{e}_{\mathrm{UV}}$ used for the first irradiation. The result of such an experiment is displayed in Fig. 7. A film with previously recorded dichroism as was shown in Fig. 6 was installed in a new position at $\varphi$ $=-30^{\circ}\left(\right.$ or $\left.150^{\circ}\right)$ and irradiated by $s$-polarized UV light. The absorption spectra taken afterward for the two positions of the sample perpendicular to each other $\left(150^{\circ}\right.$ and $\left.60^{\circ}\right)$ are presented in the main plot of Fig. 7 and the angular dependence of absorbance $(A)$ is shown in the inset for the mesogenic band $(244 \mathrm{~nm})$ and the trans band of the azochromophore $(348 \mathrm{~nm})$. The induced orientational order parameter $S=(N-1) /(N+1)$ estimated from the dichroic ratio $N=A\left(60^{\circ}\right) / A\left(150^{\circ}\right)$ is higher for the azochromophores $(S=0.45)$ than for the mesogenic groups $(S=0.24)$. In any case, it is high enough to provide an easily controllable, polarization sensitive, and reversible recording of holographic information.

\section{Photoinduced optical anisotropy}

For copolymer (I) some data on photoinduced anisotropy and phase changes may be found in Ref. 18. Here, we focus more on copolymer (II) manifesting a stable dichroism. The induced anisotropy was studied in a $10 \mu \mathrm{m}$ thick cell made of two glass plates. A phase transition was observed at $74.8^{\circ} \mathrm{C}$ as a very faint change of optical transmission and the occurrence of very small defects in the otherwise black cell between crossed polarizers. Below this temperature the polymer begins to show photosensitivity, in the sense that the birefringence can be induced under the action of incident polarized light from the microscope lamp. There seems to be three different resulting textures of the polymer after the phase transition: (a) if not illuminated, a slightly scattering texture appears, which is blue-green under crossed polarizers, but optically isotropic; (b) if illuminated by polarized light, the polymer becomes transparent and birefringent; and (c) if illuminated by unpolarized light the substance shows an optically isotropic, clear appearance.

Studies of the photoinduced birefringence were carried out at temperature $70{ }^{\circ} \mathrm{C}$, but the induced birefringence could be inscribed down to room temperature, with successively longer times needed for creating a certain optical contrast. An achieved level of the photoinduced retardation, for instance, that corresponds to a $\lambda / 2$ plate, was observed to be stable over, at least, several days. By increasing the temperature up to $80^{\circ} \mathrm{C}$, the inscribed birefringence could be quickly erased. After cooling the sample below the transition at $74.8^{\circ} \mathrm{C}$, the birefringence could be induced again by polarized white light from a microscope lamp. By following the growth of the birefringence for $30 \mathrm{~min}$, during exposure to polarized light at temperature $70{ }^{\circ} \mathrm{C}$, it was possible to estimate the induced retardation. By inserting compensating $\lambda / 4$ and $\lambda$ plates, a quantitative measure of the birefringence could be obtained. In about $5 \mathrm{~min}, \lambda / 4$ is reached, and then it takes more than $20 \mathrm{~min}$ to reach $\lambda$. After $30 \mathrm{~min}, 5 \lambda / 4 \mathrm{re}-$ tardation was obtained. The process seems to saturate around this value for the present polymer. With a $10 \mu \mathrm{m}$ cell, a retardation of $5 \lambda / 4$ corresponds to an induced birefringence of about 0.06. By irradiating the sample with unpolarized light, an inscribed in-plane birefringence could be erased, most probably, by reorientation of the optic axis into a homeotropic state. Both the homeotropic and a birefringent state could be inscribed on the optically isotropic phase at lower temperatures.

All these observations are consistent with the photoinduced mesogenic group orientation observed for much thinner films and discussed above. In a separate experiment we have checked that the white, nonfiltered light of the metalhalide lamp used in our photochromism studies produces a small excess of cis isomers with respect to the equilibrium state. Hence, the cause of the anisotropy induced by white light may easily be understood. However, the optical anisotropy might also be induced by solely green and even yellow light, at least, for a longer time illumination. Our spectroscopic experiments show an evidence that green or yellow light may produce an excess of only trans isomers. Therefore, in order to explain the effect of the photoinduced anisotropy in this case, we have to imagine that a long-wave light creates trans and cis isomers simultaneously due to some overlap of the trans $\pi-\pi^{*}$ and cis $n-\pi^{*}$ bands with some excess of trans isomers. Then, the creation of cis iso- 
mers could be a driving force for the mesogenic group orientation as was discussed above. For example, with an Arlaser excitation one could start with overwhelming amount of trans isomers and finish the process of reorientation with a substance even more enriched by trans isomers with the whole process driven by the cis-isomers excitation. Evidently, such a multistep process of the optical anisotropy recording, which involves a competitive excitation of both isomers takes a much longer time than a selective formation of either cis or trans long-living isomers by properly chosen light wavelengths, as suggested here.

\section{CONCLUSION}

Photochromism has been studied on two comb-like liquid-crystalline copolymers (I) and (II) containing mesogenic units and azobenzene chromophores in their side chains. The two copolymers possess a smectic ordering in their glassy state and manifest a strong photoinduced optical anisotropy that is of great interest for holographic information recording. In both polymers, upon short-time irradiation by unpolarized UV light, long-living (in darkness) cis isomers are observed in both chloroform solutions and spincoated films. The isomers can easily be converted into their trans counterparts by short-time yellow light irradiation. Despite such a similarity, the physical mechanisms of the photoinduced anisotropy in the two copolymers seem to be quite different. In spin-coated films of polymer (II) the origin of the anisotropy is a strong dichroism, stable, at least, for many days, which can be induced very rapidly in two steps, namely, by subsequent irradiation of the films by UV light linearly polarized in a certain direction and yellow light linearly polarized in the perpendicular direction. This dichroism occurs due to an enrichment and depletion of the chosen angular direction, correspondingly, with trans and cis isomers of the azobenzene chromophores. Polymer (I) manifests no dichroism at all, and its induced optical anisotropy may be accounted for by the known mechanism of rather slow chromophore reorientation. The detail spectral study of copolymer (II) shows that, simultaneously with the induced dichroism, a considerable reorientation of the mesogenic groups occurs as a secondary phenomenon at the stage of the cis-isomer formation. The formation of trans isomers is accompanied by a weak back-reorientation process. This observation helps us to understand better the general process of the light-induced molecular reorientation in polymers, liquid crystals, and Langmuir-Blodgett films.

\section{ACKNOWLEDGMENTS}

This work has partially been carried out in the framework of an INTAS-98 grant and was supported by a Grantin-Aid for Scientific Research from the Ministry of Education, Science, Sports and Culture (Japan). One of the authors (L.M.B.) thanks the Japanese Society for Promotion of Science for a fellowship at the Osaka University.

${ }^{1}$ Photochromism, Molecules and Systems, edited by H. Durr (Elsevier, New York, 1990).

${ }^{2}$ M. Irie, Jpn. J. Appl. Phys., Part 1 28, 215 (1989).

${ }^{3}$ T. Todorov, L. Nikolova, and N. Tomova, Appl. Opt. 23, 4309 (1984).

${ }^{4}$ J. H. Wendorf and M. Eich, Mol. Cryst. Liq. Cryst. 169, 133 (1989).

${ }^{5}$ D. Y. Kim, S. K. Tripathy, L. Li, and J. Kumar, Appl. Phys. Lett. 66, 1166 (1995).

${ }^{6}$ N. Tanio and M. Irie, Jpn. J. Appl. Phys., Part 1 33, 1550 (1994).

${ }^{7}$ T. Fischer, L. Läsker, J. Stumpe, and S. G. Kostromin, Photochem. Photobiol. 80, 453 (1994).

${ }^{8}$ N. C. R. Holme, P. S. Ramanujam, and S. Hvilsted, Appl. Opt. 35, 4622 (1996).

${ }^{9}$ T. G. Pedersen and P. M. Johansen, Phys. Rev. Lett. 79, 2470 (1997).

${ }^{10}$ Z. Sekkat and M. Dumont, Appl. Phys. B: Photophys. Laser Chem. 54, 486 (1992).

${ }^{11}$ L. M. Blinov, M. I. Barnik, T. Weyrauch, S. P. Palto, A. A. Tevosov, and W. Haase, Mol. Mater. 5, 311 (1995).

${ }^{12}$ M. I. Barnik, V. M. Kozenkov, N. M. Shtykov, S. P. Palto, and S. G. Yudin, J. Mol. Electron. 5, 53 (1989).

${ }^{13}$ S. P. Palto, L. M. Blinov, S. G. Yudin, G. Grewer, M. Schönhoff, and M. Lösche, Chem. Phys. Lett. 202, 308 (1993).

${ }^{14}$ L. M. Blinov, J. Nonlinear Opt. Phys. Mater. 5, 165 (1996).

${ }^{15}$ E. Sackman, J. Chem. Soc. A 93, 7088 (1971).

${ }^{16}$ B. Schnuriger and J. Bourdon, J. Chim. Phys. Phys.-Chim. Biol. 73, 795 (1976).

${ }^{17}$ A. Petri, S. Kummer, H. Anneser, F. Feiner, and C. Bräuchle, Ber. Bunsenges. Phys. Chem. 97, 1281 (1993).

${ }^{18}$ M. V. Kozlovsky, V. P. Shibaev, A. I. Stakhanov, T. Weyrauch, and W. Haase, Liq. Cryst. (in press).

${ }^{19}$ K. Anderle, R. Birenheide, M. Eich, and J. H. Wendorff, Makromol. Chem., Rapid Commun. 10, 477 (1989).

${ }^{20}$ L. Bata, K. Fodor-Csorba, J. Szabon, M. V. Kozlovsky, and S. Holly, Ferroelectrics 122, 149 (1991).

${ }^{21}$ S. G. Kostromin, A. I. Stakhanov, and V. P. Shibaev, Polymer Science, Ser. A 38, 1030 (1996).

${ }^{22}$ K. Skarp, K. Nakayama, M. Ozaki, K. Yoshino, M. Svensson, and B. Helgee (unpublished)

${ }^{23}$ E. Demikhov and M. V. Kozlovsky, Liq. Cryst. 18, 911 (1995).

${ }^{24}$ M. Shimomura and T. Kunitake, J. Am. Chem. Soc. 109, 5175 (1987).

${ }^{25}$ L. M. Blinov, M. V. Kozlovsky, M. Ozaki, and K. Yoshino, Mol. Mater. 6, 235 (1996).

${ }^{26}$ S. P. Palto and G. Durand, J. Phys. II 5, 963 (1995). 